\title{
La Evaluación. Una mirada desde América Latina y el Caribe
}

\author{
Evaluation of Public Policies. An appraisal from Latin America and the Caribbean
}

\author{
Arlette Pichardo Muñiz \\ Catedrática de la Universidad Nacional \\ de Costa Rica donde se desempeña \\ como Investigadora-Docente del \\ Centro Internacional de Política \\ Económica (CINPE). \\ Especialista en Planificación, Evaluación \\ y Programa Social. Sus libros han sido \\ publicados en Costa Rica, Argentina, \\ República Dominicana, México \\ y Estados Unidos. \\ arlettepichardomuniz@una.cr
}

Fecha de recepción:

30.1.18

Fecha de aprobación:

16.5.18

\section{Resumen}

En las últimas décadas en América Latina y el Caribe emerge un marcado interés por la evaluación de y desde la gestión pública. No obstante, el devenir de la práctica de la evaluación discurre desde hace más de medio siglo como una especie de eslabón invisible, emergiendo o "sumergiéndose" de distintas maneras sin que, hasta el momento, se avizore una sincronía de condiciones requeridas (voluntad política, capacidad técnica, desarrollo institucional y otras) para comprometer su visibilidad de manera sostenida. En este artículo, se identifican, caracterizan y sistematizan cursos de acción o recorridos, con sus respectivos itinerarios e intersecciones, que permiten decantar características o atributos de modelos de evaluación, desde los cuales se derivan las principales conceptualizaciones que permiten entender las prácticas y pautas más destacadas en los patrones de evaluación vigentes en el imaginario institucional y societario. El artículo tiene como propósito principal llamar la atención sobre los contenidos y formas de expresión de tales patrones y su herencia subsistente, ya que no siempre se tiene conciencia de ello, lo que se traduce en yuxtaposición o superposición de enfoques, métodos e indicadores de evaluación. Situación ésta que incide en las posibilidades de fortalecimiento y consolidación de una cultura de evaluación como propósito de futuro y de largo alcance. 
Palabras Clave: evaluación - gestión pública políticas públicas - América Latina - Caribe

\begin{abstract}
During the last decades, in Latin America and the Caribbean there has been a growing interest in the evaluation of, and from, public management. Its apparent novelty notwithstanding, in real life appraisal methods have been emerging or "submerging" in various ways for more than half a century although without, up to now, an observation of the synchrony of required conditions (political will, technical capacity, institutional development, among others) that compromise its visibility in a sustained manner. In this article, six courses of action or routes are identified, characterized, and arranged, with their respective itineraries and intersections, in order to identify models of evaluation in order to achieve an understanding of the practice and the most outstanding guidelines in the assessment patterns in the institutional and societal imaginary. The main purpose of the article is to draw attention to the contents and forms of expression of such patterns of evaluation and their persistent heritage from previous conceptual models. Unawareness of such conceptual continuity frequently leads to an overlapping of theoretical approaches, methods, and assessment indicators - a situation reducing the possibilities of strengthening and consolidating a professional culture of policy-evaluation as a long-term and far-reaching purpose.
\end{abstract}

Key-words: policy-evaluation - public management -public policies - Latin America - the Caribbean.

\title{
Introducción ${ }^{1}$
}

En América Latina y el Caribe desde la década de 1990 y en lo que va del siglo xxi, el aparato estatal empieza a reasumir, en alguna medida, funciones que le habían sido propias y a colocar otras en su agenda, incluida la reforma y modernización del Estado: “El Estado está de moda, y con él, las políticas públicas” (Fontaine 2015:1). 
Desde el punto de vista de la evaluación, la novedad en la moda del Estado y de las políticas públicas es que tímidamente se comienza a cobrar conciencia de su necesidad e importancia, y en cierto modo se vislumbra algún interés por ubicar a la evaluación dentro de los ámbitos de estudio de las políticas públicas (donde se registra la mayor ausencia), más allá de la práctica convencional de la evaluación de proyectos y programas con miras a valorar, generalmente en forma aislada, el cumplimiento de metas preestablecidas.

De acuerdo a Fontaine "La institucionalización del estudio de las políticas públicas permitió la emergencia de nuevas teorías y el perfeccionamiento de los métodos de análisis" (loc.cit.). Cabría preguntarse, no obstante, si en América Latina y el Caribe la institucionalización del estudio de la evaluación está permitiendo/promoviendo/facilitando la emergencia de nuevas teorías y el perfeccionamiento de los métodos de evaluación. En general, es posible argumentar que la evaluación ha discurrido como una especie de eslabón invisible, vale decir ‘que no puede ser visto' o ‘que rehúye ser visto' (Real Academia Española 2017). En ese sentido, "emerge" o se "sumerge" según el país y momento histórico que se trate, atendiendo a la coincidencia entre factores vinculados con la voluntad política, las capacidades institucionales, el desarrollo de habilidades técnicas, entre otros factores.

La conceptualización de la evaluación, así como los enfoques, métodos e indicadores a su alcance desde sus incursiones iniciales provienen, en general, de la evaluación de la educación, beneficiándose de sus potencialidades (en particular el desarrollo instrumental), como también arrastrando su principal limitación (percepción de amenaza y castigo o penalización). Con el tiempo, sin perder de vista su origen, el interés por la evaluación comienza a anclarse en las experiencias de planificación nacional y otras iniciativas vinculadas a la formulación de programas o proyectos. Por lo regular, dicho asocio adquiere características particulares en función del contexto sociopolítico, incluyendo las características de los Estados y sus relaciones con la sociedad civil (Neirotti 2012). Consustancial a la vinculación con la planificación, empieza a brillar la ausencia de ejecutoria, salvo aquellas iniciativas financiadas por la cooperación internacional o fuertemente arraigadas en el padrinazgo de actores nacionales de peso en la toma de decisiones institucionales.

En este marco brevemente descrito, cabría preguntarse ¿será que en el momento actual el sentido u orientación de la evaluación está empezando a cobrar un posicionamiento estratégico que puede favorecer/promover/facilitar su visibilización en la agenda de las políticas públicas?Y, si así fuera, también cabría preguntar: ¿será que, finalmente, la intención o disposición de la evaluación se colocará en el lugar que le corresponde, más allá del aspecto normativo (insumo para la toma de decisiones y generación de medidas preventivas y correctivas) en tanto actitud ante las cosas y ante la vida, instrumento para construir el futuro? (Pichardo Muñiz 1993). O bien, por el contrario, ¿será que la evaluación al asumirse de y desde la gestión pública pasará a formar parte del conjunto de actividades rutinarias con escasa relevancia o nula incidencia en la agenda política, técnica e institucional? He ahí un dilema con implicaciones y repercusiones insospechables. 
En procura de búsqueda de respuestas a esas y otras interrogantes, y sin ánimo de llegar a conclusiones irrefutables, el objeto central del presente artículo es identificar, caracterizar y sistematizar, en grandes rasgos, seis cursos de acción o recorridos, con sus respectivos itinerarios e intersecciones, que permiten decantar características o atributos de modelos de evaluación, desde los cuales se derivan las principales conceptualizaciones para entender las prácticas y pautas más destacadas en los patrones de evaluación vigentes en el imaginario institucional y societario.

\section{Diagrama 1: Lógica de argumentación del estudio}

\section{CURSOS DE ACCIÓN O RECORRIDOS}

(itinerarios e interacciones)

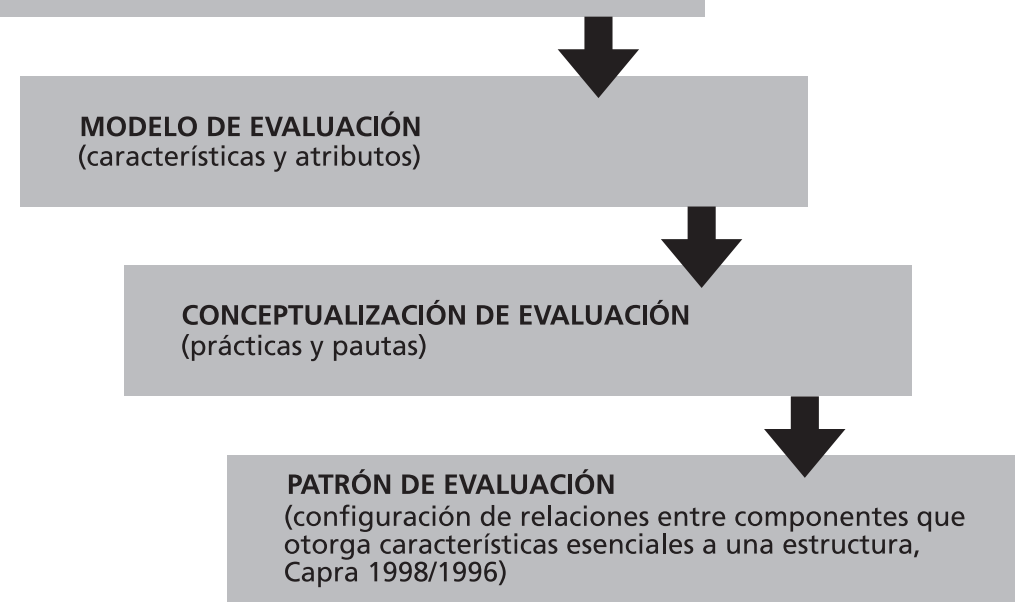

Fuente: Elaboración propia con base en experiencias propias

El artículo tiene como propósito principal llamar la atención sobre los contenidos y formas de expresión de tales patrones y su herencia subsistente, ya que no siempre se tiene conciencia de ello, lo que se traduce en yuxtaposición o superposición de enfoques, métodos e indicadores de evaluación. Situación ésta que incide en las posibilidades de fortalecimiento y consolidación de una cultura de evaluación en la gestión pública como propósito de futuro y de largo alcance. En última instancia, se trata -en la medida de lo posible- de deslindar aprendizajes que permitan plantear algunas coordenadas básicas con la intención de compartir, crear y re/crear una especie de hoja de ruta, de cara a contribuir con el fortalecimiento y consolidación de una cultura de evaluación.

El estudio se basa en un recuento de literatura especializada. La investigación documental brinda soporte de los puntos de vista que se presentan y los argumentos que se desarrollan. Asimismo, se recurre a conocimiento de primera mano: quien esto escribe ha 
sido parte consustancial de los variados cursos de acción o recorridos que se identifican, de manera que este texto parte del pensar y sentir de la experiencia propia.

El artículo se organiza de la siguiente manera. En la primera sección se identifica en términos generales un conjunto de cursos de acción o recorridos de la evaluación en América Latina y el Caribe. En las secciones siguientes se exponen los itinerarios y posibles interacciones de los cursos de acción o recorridos identificados, con base en una breve contextualización a partir de una sucinta relación de hechos de interés; asimismo se sistematizan las características y atributos de los modelos de evaluación en que devienen y las prácticas y pautas que impregnan la conceptualización de la evaluación y las coordenadas básicas de la configuración de relaciones entre componentes, que le otorga características esenciales a la estructura de los patrones de evaluación vigentes. Se concluye con algunas reflexiones a manera de lecciones a tener en cuenta en el accionar de la evaluación, particularmente en la Gestión Pública.

\section{De los cursos de acción o recorridos ${ }^{2}$}

Es posible asociar el interés primario por la evaluación en América Latina y el Caribe hacia la década de 1950 a las primeras iniciativas de planificación nacional en el continente, bajo la influencia de la CEPAL (Comisión Económica para América Latina y el Caribe, de la ONU), en el marco de los objetivos del modelo de desarrollo hacia adentro vía la industrialización sustitutiva de importaciones. A partir de 1961 tal interés se engarza en el ámbito de las experiencias de planificación nacional surgidas al calor de la Alianza para el Progreso, en particular en los países de Centroamérica y el Caribe. De esta manera la evaluación empieza a extenderse hacia otros ámbitos de acción gubernamental y no gubernamental, para incluir a los denominados aspectos sociales del desarrollo (en especial educación, salud y saneamiento ambiental, vivienda y control de la natalidad) que circundan a las propuestas de reformas sociales suscritas en la Carta de Punta del Este (Pichardo Muñiz 1984).

A partir de la década de 1980, particularmente en Centroamérica, en el marco de la ayuda humanitaria primero y la cooperación para el desarrollo después, la tendencia es impulsar iniciativas de evaluación de proyectos y programas ejecutados por instancias de la sociedad civil, aunque también con la participación de los gobiernos nacionales, centrados -en lo fundamental- en la valoración de la pertinencia, la eficacia y eficiencia institucionales. Desde mediados de la década de 1990 en adelante, la impronta de la evaluación, aunque sigue procediendo de la cooperación internacional, recibe un fuerte influjo de manera particular desde programas institucionales bajo el alero de la Protección Social, que incluso empiezan a incorporar la evaluación desde la formulación o diseño inicial de los programas y a acudir a instrumental estadístico sofisticado. En forma relativamente simultánea a lo anterior, y con mayor fuerza durante el siglo XXI, se inicia el llamado movimiento de la institucionalización de los Sistemas de Monitoreo y Evaluación por medio del cual, con desigual intensidad, se busca colocar a la evaluación como parte integrante de y desde la gestión pública. 
Resumiendo, y sin desmedro de que puedan identificarse otros cursos de acción o atendiendo a propósitos diversos, para los fines de este artículo se estructuran seis recorridos básicos.

Diagrama 2: Cursos de acción o recorridos de la evaluación en América Latina y el Caribe

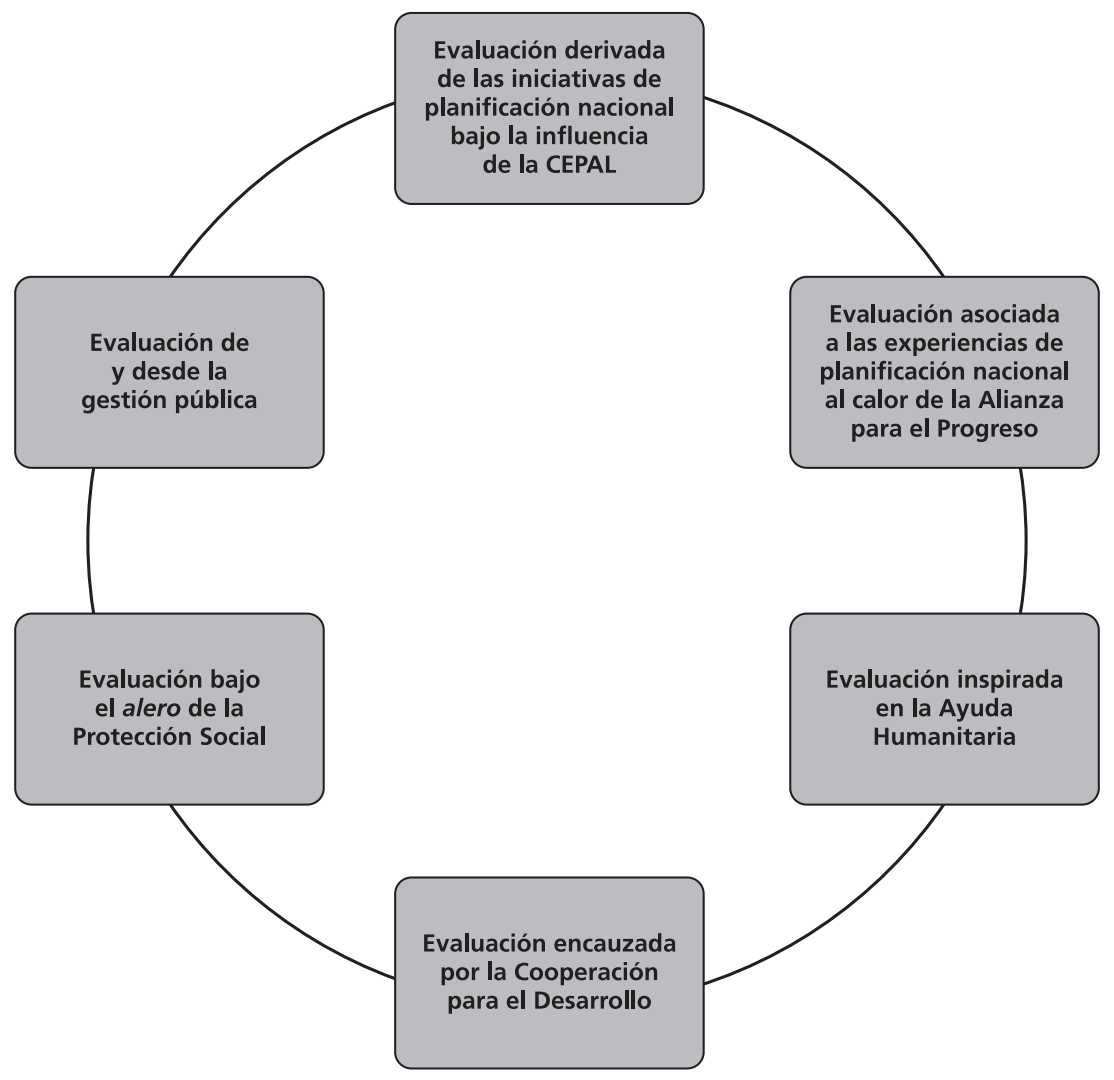

Fuente: Elaboración propia con base en experiencias previas.

Para la presentación de los cursos de acción o recorridos identificados en la evaluación en América Latina y el Caribe se recurre a la reconstrucción de hitos o sucesos históricos, tales como: i) influencia de decisiones políticas de envergadura global con impacto en la conducción de los gobiernos nacionales; ii) incidencia de organismos internacionales regionales y extra regionales, en particular su asocio con la producción de literatura especializada sobre evaluación; iii) unidad de intervención que constituye el foco y objeto de evaluación por excelencia; iv) momento de evaluación que se privilegia en relación con la ejecución (ex ante, concurrente o ex post); y v) orientación predominante en los usos de los indicadores comúnmente utilizados en la evaluación. 


\section{Evaluación derivada de las primeras iniciativas de planificación nacional bajo el impulso de la CEPAL}

La atención sistemática en la evaluación deriva inicialmente de las primeras iniciativas de planificación nacional bajo el impulso de la CEPAL, a cargo de corporaciones de fomento u otros tipos de organismos o instancias creadas para tal fin, en el marco de la consolidación del Estado interventor en la economía. Tales iniciativas emergen del modelo de desarrollo hacia adentro vía la industrialización sustitutiva, generalmente asociado a la figura del maestro Raúl Prebisch (Prebisch 1963). Fernando Henrique Cardoso (1977) refiere esta propuesta como "la originalidad de la copia: la CEPAL y la idea del desarrollo".

Las características esenciales de la planificación normativa que impregnan a la conceptualización y práctica de la evaluación, se pueden sintetizar de la siguiente manera: la velocidad del crecimiento económico constituye el principal foco de interés, la planificación global el enfoque preferencial, el mediano plazo el horizonte temporal por excelencia, el Plan Nacional de Desarrollo la expresión máxima en tanto dispositivo de la planificación nacional, formulado con predominancia de la disciplina económica; y, por tanto, centrado en instrumental de análisis de dicha disciplina (modelo de insumo-producto, programación lineal, modelos de equilibrio general y de simulación económica) (Pichardo Muñiz 2013).

El enfoque hacia la atención de obstáculos o restricciones para el desarrollo (suministro de energía, infraestructura para la provisión de transporte y comunicaciones y mejoramiento de las condiciones sanitarias de la población) lleva a que, consecuentemente, se le asigne a la evaluación el papel de valorar ex ante, como parte de los estudios de factibilidad, a proyectos en sí mismos diseñados para resolver tales problemas. Desde esa perspectiva, la evaluación tiende a circunscribirse en el ámbito de proyectos de infraestructura de apoyo a la política de desarrollo industrial, en particular aquellos en manos de Corporaciones de Fomento que, en su momento, constituyen un ícono en materia de evaluación (Pichardo Muñiz 1993).

Este modelo de evaluación deriva en prácticas y pautas cuyo principal propósito se centra en valorar el riesgo de la inversión (ventajas y desventajas) para establecer prioridades de ejecución, con base en el principio de escasez proveniente de la economía clásica: la limitación de recursos frente a las necesidades infinitas o ilimitadas (Pichardo Muñiz, 2017). Su principal sustento bibliográfico se recoge en el Manual de Proyectos de Desarrollo Económico (CEPAL 1958), obra pionera en la materia. Sus contenidos son retomados por el Instituto Latinoamericano de Planificación Económica y Social (ILPES) desde su creación a principios de la década de 1960, en tanto brazo ejecutor de la CEPAL en materia de formación en planificación, economía y gestión del sector público para los países de América Latina y el Caribe. La Guía para la presentación de proyectos da cuenta de ello, elaborada con la participación de otros organismos internacionales y de la cooperación del Consejo Federal de Inversiones de la Argentina, la Corporación 
de Fomento de Chile y la Oficina de Coordinación y Planificación (CORDIPLAN) de la Presidencia de Venezuela (ILPES 1973 y reediciones posteriores).

La Guía citada arriba incluye un capítulo específico, con su respectivo anexo, bajo la denominación de "Evaluación Económica", que parte del siguiente concepto de evaluación:

(...) constituye un balance de las ventajas y desventajas de asignar al proyecto analizado los recursos necesarios para su realización. En términos más amplios, la tarea de evaluar consiste en comparar los beneficios y los costos del proyecto, con miras a determinar si el cociente que expresa la relación entre unos y otros presenta o no ventajas mayores que las que se obtendrían con proyectos distintos, igualmente viable. Enfocando aun desde un tercer ángulo la evaluación como elemento de juicio sobre un proyecto, se trata de determinar si el aporte del proyecto a los objetivos del desarrollo económico $y$ social justifica su realización, teniendo en cuenta los usos alternativos que pueden tener los mismos recursos. En suma, se podría decir que en la evaluación económica se aplican ciertos criterios preestablecidos al análisis de los resultados netos del proyecto para decidir si es viable, conveniente y oportuno realizarlo (ILPES, 1973:137; destacados agregados).

En buenas cuentas, en este marco la evaluación de proyectos trata del análisis de la rentabilidad del capital a precios de mercado. En consecuencia, los principales indicadores en juego son la Tasa Interna de Retorno (TIR) y elValor Actualizado (VAN), así como indicadores financieros (Pichardo Muñiz 1993).

Un enfoque de evaluación de esa naturaleza presenta una serie de limitaciones, cuya consideración rebasa los límites del presente artículo. No obstante es importante señalar que, por un lado, los propósitos del desarrollo humano sostenible no siempre son compatibles con la rentabilidad del capital; por el otro, los precios de mercado pueden ser representativos del valor real de los bienes y servicios, en el caso que las reglas de la oferta y la demanda pudiesen funcionar en condiciones de competencia "perfecta", ocupación plena de recursos y movilidad de los factores de producción. En otro orden, los beneficios derivados de los proyectos no siempre son susceptibles de ser expresados fácilmente en forma numérica (Pichardo Muñiz 1993).

Del sustrato de esta perspectiva de evaluación se deriva lo que se conoce como el enfoque de evaluación "social", cuyos fundamentos no son radicalmente distintos, aunque difieren 
en que sustituye o complementa el análisis de rentabilidad en función de ciertos parámetros de interés nacional. Razón por la que debería denominarse evaluación económica nacional de proyectos como sugieren Cohen y Franco (1992: 83). Al respecto esos autores indican:

(...) utiliza las herramientas del análisis económico para comparar los costos y beneficios, dentro del ámbito de una sociedad determinada. Al priorizar las contribuciones que distintos proyectos hacen a la sociedad como un todo, la evaluación social racionaliza la toma de decisiones en materia de inversión pública.

La mayor diferencia de la llamada Evaluación Social de Proyectos radica que en lugar de acudir directamente a los precios de mercado, se maneja con lo que se conoce como precios sociales o precios sombra (también conocidos como de cuenta o de eficiencia), con el propósito de corregir las imperfecciones del mercado (Pichardo Muñiz 1993).

Resumiendo: la evaluación derivada de las primeras experiencias de planificación nacional bajo la influencia de la CEPAL, deviene un modelo de evaluación cuyas características y pautas principales es posible enunciar de la manera siguiente:

- Centrada en el proyecto en sí mismo, en tanto unidad de intervención;

- Circunscrita a la evaluación ex ante, previa a la decisión de su ejecución, como parte de los estudios de factibilidad;

- Fundamentada en indicadores de naturaleza económico-financiera.

El patrón de evaluación, consistente en la conceptualización adoptada de la evaluación como elemento de juicio de un proyecto y su aporte a los objetivos nacionales de desarrollo, tiene como propósito expreso establecer prioridades de inversión, de cara al uso alternativo de recursos de inversión (privada o pública).

\section{Evaluación asociada a las experiencias de planificación nacional al calor de la Alianza para el Progreso}

Al calor de la Alianza para el Progreso, una iniciativa de los Estados Unidos de América (EUA) lanzada con el propósito de contrarrestar posibles influencias de la revolución cubana, la Carta de Punta del Este, suscrita en Uruguay, en 1961, por los países integrantes de la Organización de los Estados Americanos (OEA), con excepción de Cuba, se comprometen a llevar adelante un conjunto de reformas sociales estructurales, en el marco de fortalecer la democracia y salvaguardar la libre empresa, como condición para el otorgamiento de 
donaciones y la concesión de préstamos. En la práctica, las principales intervenciones, entre otras medidas, se traducen en campañas masivas y a nivel nacional de alfabetización y saneamiento ambiental, repartos de tierra para uso agrícola, construcción de viviendas de interés social, programas de control de la natalidad (éstos últimos en su momento se convirtieron en íconos en materia de evaluación e investigación) (Pichardo Muñiz 1984).

En ese marco se crean Oficinas Nacionales de Planificación en Centroamérica y el Caribe (Costa Rica, República Dominicana, Jamaica, para mencionar algunos países) y se fortalecen e institucionalizan las primeras experiencias de planificación nacional ya existentes (México, Argentina, Brasil, Chile, entre otros). Consustancial a su origen, estas instancias de planificación nacional centralizan la aprobación de la cooperación internacional, función que mantienen hasta el presente en la mayoría de los países.

Sin perder su enfoque normativo (más bien en algunos países se profundiza), la función de planificación nacional empieza a adquirir características distintas a las otorgadas en el contexto de la política de industrialización sustitutiva, que dejan su huella en la orientación de las experiencias de evaluación. En esta oportunidad, interesa la dirección del desarrollo más que la velocidad del crecimiento económico. De ahí que el desarrollo económico per se deja de ser el único centro de atención y se empieza a enfatizar en los llamados aspectos sociales del desarrollo, aunque sin lograr la debida articulación. El enfoque preferencial de la planificación global pasa a incorporar a la planificación sectorial y el horizonte temporal del mediano plazo se expresa también en el corto plazo. El Plan Nacional de Desarrollo continúa siendo el dispositivo por excelencia, pero se incorporan acciones concretas, incluso llegando a convertirse tales planes en una especie de sumatoria de programas y proyectos (Pichardo Muñiz 1984).

La evaluación cobra un giro diferente, desde la perspectiva de los procesos de reformas sociales, con la inclusión de los llamados aspectos sociales del desarrollo y el énfasis en campañas nacionales con propósitos de mejoramiento de la condición y calidad de vida de amplios sectores de la población. En lugar de centrarse en valorar la asignación de recursos previo a la ejecución, pasa a ocuparse del alcance o determinación de cobertura de los programas ejecutados. De igual modo, más allá de los indicadores económicos y financieros usualmente utilizados en la evaluación económica de proyectos, se comienza a incursionar en los llamados indicadores sociales. Cabe resultar que, en ese sentido, la tendencia más socorrida es la recurrencia a listados de indicadores "prefabricados" cuya utilización se propicia en ocasiones carentes de contexto e integralidad.

De alguna manera, aunque con alcance limitado en términos de un amplio y sostenible impacto institucional, se empieza a observar una cierta influencia del llamado movimiento de los indicadores sociales (Bauer 1996) que surge en cuestionamiento a los usos del Producto Interno Bruto (PIB) como indicador per se de desarrollo. Destacan, asimismo, los usos de la investigación evaluativa (Weiss 1978). 
Con el tiempo este enfoque de evaluación, con fuerte influencia de la academia norteamericana especialmente proveniente de las escuelas de administración, se enraíza en el accionar del sector público, quizás por su simpleza, caracterizando al ejercicio evaluativo, cuando se practica, y constituyéndose en una impronta recuperada y adoptada por los organismos de control, al velar por la observancia irrestricta de normativas y procedimientos, en la mayoría de los casos sin tomar en cuenta la creación de condiciones institucionales para la viabilidad de las intervenciones.

Desde esta perspectiva se incita a la medición cuantitativa de resultados obtenidos, utilizando indicadores que dan cuenta de manera particular de tres aspectos básicos, a saber:

I. Volumen de Trabajo, cantidad de actividades realizadas para el logro de resultados previstos. Por ejemplo, número de lecciones impartidas, cantidad de horas de clases, cantidad de material educativo producido y distribuido, etc.

II. Producto Final, cantidad de bienes producidos o servicios ofrecidos. Por ejemplo: número de estudiantes atendidos.

III. Realizaciones, resultados o productos obtenidos.

Por ejemplo: cantidad de estudiantes promovidos (Pichardo Muñiz 1993).

En resumen, la evaluación asociada en las experiencias de planificación nacional al calor de la Alianza para el Progreso, en el marco de propuestas de reformas sociales estructurales, deviene en un modelo de evaluación, cuyas características y pautas principales varían en relación con el curso de acción anterior al centrarse en:

- El programa en tanto unidad de intervención;

- Tendencia a "mover" el interés de la evaluación al momento ex post, centrada en el propósito de valorar los alcances es términos de cobertura;

- Consecuentemente, el sustento de indicadores producto de la cuantificación, para dar cuenta del volumen de trabajo, la cantidad de producto final y las realizaciones.

En este contexto el patrón de evaluación pasa a ocuparse más de la cuantificación de metas que de la contribución de las intervenciones a los objetivos nacionales de desarrollo, siendo la calidad de las intervenciones y sus efectos e impactos en la población destinataria la gran ausente en las experiencias de evaluación de ese momento. 


\section{Evaluación inspirada en la ayuda humanitaria}

El debilitamiento y descrédito de las funciones del Estado en América Latina y el Caribe y la búsqueda de racionalización del llamado gasto público, particularmente post crisis y durante el ajuste estructural, llevan a una preponderancia de instancias de la sociedad civil en la ejecutoria de intervenciones para la prestación de servicios, en ámbitos propios de funciones del Estado. De manera particular en Centroamérica, con los conflictos bélicos como telón de fondo, sus impactos en la población desplazada, refugiada y retornada, y el interés en la firma de acuerdos de paz.

La ayuda humanitaria proveniente de Estados Unidos, Canadá y Europa, canalizada por medio de entidades para tal fin, como Agencias de Estados (el caso de la Agencia de los Estados Unidos para el Desarrollo, USAID), universidades, iglesias y otras entidades inspira una forma particular de hacer evaluación, orientada fundamentalmente hacia la fiscalización del cumplimiento de objetivos y metas y la transparencia en el uso y manejo de los recursos financieros, convirtiendo a las Organizaciones No Gubernamentales (ONG) en íconos en materia de evaluación.

Esta perspectiva trae de vuelta al proyecto como objeto central de evaluación; aunque continúa centrada en lo fundamental en la evaluación ex post, tiende a focalizarse, más allá de la cobertura alcanzada, en el cumplimiento de los propósitos iniciales generadores de la intervención y en la recuperación de experiencias para otros proyectos, otros sectores, otros grupos de interés, otros países, otros continentes.

En esta oportunidad empieza a prevalecer, de manera preferencial, el examen o la verificación del nivel de cumplimiento de las metas alcanzadas versus las metas programadas, en ocasiones con matices de productividad, bajo criterios de eficacia y eficiencia. Marco en el cual la evaluación intermedia o de progreso comienza a abrirse paso, generalmente practicada en un punto intermedio del horizonte temporal de las intervenciones con el propósito de detectar ajustes. De igual forma, se desarrolla un interés por conocer la opinión de la población destinataria, generalmente como fuente de información y a menudo sin criterios claros de escogencia y representatividad.

En correspondencia con el curso de acción anterior, se destaca el énfasis en hacer de la evaluación un ejercicio de comparación entre la ejecución (lo efectivamente realizado) y la programación (lo propuesto inicialmente), aplicando medidas de estadística descriptiva para realizar valoraciones en términos cuantitativos. De manera que tanto la lógica del procedimiento evaluativo como de los indicadores utilizados para tal fin, se desprenden de la comparación entre la planificación y el cumplimiento de la misma.

Resumiendo: con la evaluación inspirada en la ayuda humanitaria, en el marco del debilitamiento y descrédito de las funciones del Estado, y en Centroamérica de los conflictos políticos, se impulsa un modelo de evaluación con prácticas y pautas orientadas en lo fundamental a: 
- Renovación del interés en el proyecto en tanto unidad de intervención, aunque no así la conceptualización de la evaluación como instrumento para medir su contribución a objetivos nacionales de desarrollo;

- Énfasis aún mayor en objetivos cuantitativos, interesando como propósito central realizar comparaciones entre lo programado inicialmente y lo efectivamente realizado, irrumpiendo con fuerza el uso de medidas de estadística descriptiva;

- Incorporación, como fuente de información, de la población destinataria de las acciones evaluadas;

- De cara a la recuperación de experiencias para futuras actuaciones, se comienzan a valorar criterios de evaluación más allá de los alcances en términos de cobertura, especialmente vinculados a la opinión de la población destinataria. - El patrón de evaluación se configura con orientación generalizada hacia la fiscalización del cumplimiento de metas y el manejo de los recursos financieros.

\section{Evaluación encauzada por la Cooperación para el Desarrollo}

Tras los embates de la crisis económico-financiera de finales de la década de 1970 y principios de 1980 con la deuda externa como detonante, la acción generalizada de las políticas de estabilización financiera y los programas de ajuste estructural en el marco de las imposiciones del Fondo Monetario Internacional (FMI), la liberalización financiera, la apertura comercial y la desregulación de los mercados y otras medidas provenientes del denominado consenso de Washington, se impulsan procesos de reforma y modernización del Estado, en particular bajo el enfoque gerencial (el New Public Management) que implica la adopción de principios y criterios de mercado (tales como traslado de funciones institucionales consideradas no sustantivas a terceros, promoción de competencia entre proveedores, fijación de tasas o pago por servicios entre otras).

América Latina y el Caribe se convierte en un escenario privilegiado para llevar adelante una multiplicidad de intervenciones públicas de diversa naturaleza, complejidad y alcance, en particular orientadas a la búsqueda de nexos y articulaciones sectoriales y en algunos países reforma de los sistemas transversales de gestión gubernamental (planificación-presupuestocontrol). Las implicaciones en términos de inversión, provenientes de la cooperación internacional, como de recursos propios de los países, hacen reflotar el interés por la evaluación en la gestión pública, particularmente, bajo una orientación general de rendición de cuentas y recuperación de buenas prácticas, aunque no siempre teniendo en cuenta que de cara a una replicabilidad potencial de iniciativas se requiere de la existencia de condiciones previas. La Cooperación para el Desarrollo, fundamentalmente bajo el liderazgo de la Organización para la Cooperación y Desarrollo Económico (OECD, por su sigla en inglés) y la 
Comunidad Europea (CE), encauzan a la evaluación con un mayor nivel de tecnificación de los procedimientos al amparo de una fuerte propagación de directrices y manuales de evaluación mayor, si se quiere, que los alcanzados por recorridos anteriores.

Los criterios de evaluación de la OECD empiezan a popularizarse a partir de la definición de evaluación suscrita por esa entidad:

Una función que consiste en hacer una apreciación, tan sistemática y objetiva como sea posible, sobre un proyecto en curso o finalizado, un programa o conjunto de líneas de acción, su concepción, su realización y sus resultados. Se trata de determinar la pertinencia de los objetivos y su grado de realización, la eficiencia en cuanto al desarrollo, la eficacia, el impacto y la sostenibilidad. Una evaluación debe proporcionar información creible y útil que permita integrar las enseñanzas en los mecanismos de elaboración de las decisiones, tanto de los países de acogida como de los donantes (apud Comisión Europea 2002: 78. Traducción y destacados de la autora)

Como puede observarse de la definición anterior se derivan cinco criterios claves de aplicabilidad en la evaluación concurrente, ampliamente utilizados, que permean de muchas maneras la conceptualización y práctica de la evaluación desde la gestión pública: I. pertinencia, II. eficacia, III. eficiencia, IV. impacto, V. sostenibilidad.

Tabla 1: Criterios de Evaluación impulsados por la OECD.

\begin{tabular}{|l|l|}
\hline Criterios & Definición \\
\hline Pertinencia & $\begin{array}{l}\text { La adecuación de los objetivos del proyecto a los problemas que se supone se van a resolver y al entorno } \\
\text { físico y político en el que se realiza el proyecto, incluso una evaluación de la calidad de la preparación y } \\
\text { del diseño del proyecto, o sea el carácter lógico y completo del proceso de planificación del proyecto, y la } \\
\text { lógica y coherencia internas del diseño del proyecto. }\end{array}$ \\
\hline Eficacia & $\begin{array}{l}\text { El hecho de que los resultados hayan sido realizados a un coste razonable, cómo los insumos/medios han } \\
\text { sido convertidos en resultados, en términos de calidad, de cantidad y de tiempo, y la cantidad de los resul- } \\
\text { tados obtenidos. Suele requerir la comparación con enfoques alternos para conseguir los mismos resultados } \\
\text { y asegurarse de que el proceso más eficaz haya sido adoptado. }\end{array}$ \\
\hline Eficiencia & $\begin{array}{l}\text { Una evaluación de la contribución de los resultados a la consecución de la finalidad del proyecto y de la } \\
\text { manera en que las hipótesis incluyen en los logros del mismo. }\end{array}$ \\
\hline Impacto & $\begin{array}{l}\text { El efecto del proyecto en su entorno global y su contribución a los objetivos sectoriales resumidos en los } \\
\text { objetivos globales del proyecto y a los objetivos políticos más amplios de la CE. }\end{array}$ \\
\hline Sostenibilidad & $\begin{array}{l}\text { La probabilidad de mantenimiento de la serie de ventajas derivadas del proyecto, en particular, la continuación de } \\
\text { sus actividades y la consecución de resultados, principalmente en lo relativo a los factores de desarrollo del apoyo } \\
\text { político, los factores económicos y financieros, los aspectos socioculturales, la cuestión de la igualdad de sexos, la } \\
\text { idoneidad de la tecnología, los aspectos ecológicos y la capacidad institucional. }\end{array}$ \\
\hline
\end{tabular}

Fuente: Tomado de Comisión Europea, 2002: 28 (traducción propia). 
A los criterios anteriores, la Comisión Europea agrega como criterios específicos dos más: I. valor añadido de la acción comunitaria en relación con las intervenciones de los Estados miembros; II. coherencia o refuerzo mutuo, grado en que las actividades del proyecto resultan complementarias con las políticas del país asociado y las intervenciones de otros donantes.

El programa, pero también en alguna medida el proyecto, en tanto unidades básicas de intervención de la planificación, se constituyen en objeto de evaluación, que empieza a redefinirse para examinar no solamente el alcance de las acciones, sino también para tratar de determinar en forma más amplia la contribución de las intervenciones (hasta qué punto y en qué medida) a las situaciones problemáticas que se supone deben responder con su ejecución.

El monitoreo y el seguimiento (aunque a veces confundiando los términos) empiezan a abrirse paso, más allá de la evaluación final y la evaluación de progreso o intermedia. En ese marco, el ejercicio evaluativo empieza a extender su mirada hacia los resultados (y a menudo se confunden estos con productos) aunque no necesariamente a los procesos y al desempeño institucional. En ocasiones incorporando una orientación de atención al cliente, por lo que empieza a contar la satisfacción de la población destinataria, lo que lleva a incursionar en la aplicación de instrumental de investigación social de más largo alcance y de criterios de representatividad estadística en la selección de muestras de población informante (incluso se empiezan a aplicar las llamadas encuestas de satisfacción y a crear Contralorías de Servicios).

La batería de indicadores tiende a ampliarse, con interés especial en la evaluación de la calidad o certificación de normas, al estilo de la Organización Internacional para la Normalización (ISO, por su sigla en inglés), desde la perspectiva industrial, ambiental o de otra naturaleza. Asimismo, empieza a observarse una marcada orientación hacia el cumplimiento de estándares nacionales e internacionales, a menudo definidos en forma universal y generalmente vinculados con procesos de acreditación (reconocimiento de competencias), en particular de la educación superior, que da paso a la creación de instancias especializadas para tal fin.

En síntesis, la evaluación encauzada por la cooperación para el desarrollo genera un modelo con prácticas y pautas:

- Centradas en el programa en tanto unidad de intervención, aunque también en el proyecto;

- Con énfasis tanto en la evaluación ex post como en la evaluación concurrente. No obstante el monitoreo y el seguimiento comienzan a abrirse espacio;

- Con cierto interés en el desempeño institucional y organizacional, lo que incide en el ingreso de la satisfacción de la población beneficiaria, mediante el uso de instrumental de investigación social y criterios de representatividad estadística; 
- Extendiendo la mirada hacia indicadores de evaluación de calidad, especialmente en el marco de procesos de acreditación y certificación, con referencia a estándares nacionales e internacionales.

El patrón de evaluación se centra en determinar la contribución de las intervenciones enfocadas a los propósitos que las originaron, con una orientación general de rendición de cuentas y recuperación de buenas prácticas, aunque no siempre teniendo en cuenta las condiciones de replicabilidad.

\section{Evaluación bajo el alero de la Protección Social}

En América Latina y el Caribe en el marco de la recuperación de las funciones del Estado, se renueva el interés por la política social, particularmente a raíz de la Cumbre Presidencial del año 2000, con la adopción de los Objetivos de Desarrollo del Milenio (ODS).Tal interés, en contraposición con la otrora prestación de servicios (paquetes de alimentos, servicios de educación y salud, acceso a viviendas y otros ámbitos particulares destinados a la satisfacción de necesidades en sentido más amplio), se concreta en programas de Protección Social: transferencias (fundamentalmente monetarias) concebidas como subsidios al ingreso familiar, mayormente condicionadas al cumplimiento de condiciones o corresponsabilidades en el marco de sistemas de focalización a población en condición de pobreza y en situaciones especiales (Pichardo Muñiz 2014 y Pichardo Muñiz 2018, en prensa).

Esta reorientación de la política social, con el impulso de la banca multilateral, en particular el Banco Mundial, el Banco Internacional de Reconstrucción y Fomento (BIRF), el Banco Interamericano de Desarrollo (BID) y agencias del sistema de la Organización de las Naciones Unidas (ONU), tiene amplias repercusiones en la vigencia actual de la evaluación. Por un lado, denota su importancia, incluso desde el diseño mismo; al mismo tiempo, centra el objeto de la evaluación en la búsqueda de diferencias y vntre la situación "con" o "sin" intervención, con el propósito expreso de establecer comparibilidad antes y después en medición de impactos en la población destinataria sin que, necesariamente, se tomen en cuenta efectos o impactos en otros niveles. En este marco se tiende a privilegiar el programa en tanto unidad de intervención, en particular con cierto nivel de complejidad y a introducir la realización de Estudios Línea Base, mediante la aplicación de instrumental estadístico sofisticado y modelos econométricos. En ocasiones, se incursiona también en la utilización de métodos etnográficos de naturaleza cualitativa.

Al focalizar en la medición cuantitativa de impactos en la población destinataria, la evaluación bajo el alero de la protección social revitaliza el diseño y aplicación de modelos experimentales y cuasi experimentales, con o sin grupo de control, desarrollados y aplicados a principios de la década de 1960, particularmente en Estados Unidos (Campbell y Stanley, 1963; Cook y Campbell, 1979). 
En América Latina y el Caribe durante las últimas décadas se llevan a cabo un amplio conjunto de evaluaciones de impacto de programas de transferencias monetarias, que se han convertido en ícono de evaluación. Un balance de los impactos evaluados muestra usos interesantes de indicadores en materia de capacidades humanas, pobreza y desigualdad de los ingresos, consumo, generación de ingresos e inserción laboral, trabajo infantil, empoderamiento y autonomía de las mujeres (Pichardo Muñiz, 2014).

En resumen, se genera de esta manera un modelo de evaluación con prácticas y pautas centradas en:

- El programa, especialmente de gran alcance y altos niveles de compledidad, en tanto unidad de intervención.

- El interés de realizar comparaciones cuantitativas "con" o "sin" intervención.

- La realización de Estudios Línea Base para medir el antes y la evaluación de impacto para el después.

- El uso de diseños esperimentales y cuasi experimentales, con o sin grupo de control.

- Consecuentemente, la utilización de instrumental de análisis estadístico sofísticado.

El patrón de evaluación se orienta hacia la medición de efectos e impactos en la población destinataria; sin que, necesariamente, se tengan en cuenta la necesidad de evaluar en forma integral efectos e impactos en las instituciones, organizaciones y la sociedad en su conjunto.

\section{Evaluación enraizada de y desde la gestión pública en general}

En varios países de América Latina y el Caribe (por ejemplo República Dominicana, Chile, Perú entre otros) se llevan a cabo reformas sectoriales, que implican modificaciones de los sistemas transversales de la gestión pública. En ese marco, en alguna medida se revaloriza la función pública y cobra vigencia una mayor comprensión de la necesidad de evaluar las razones por las cuales algunas intervenciones públicas pueden funcionar bien y otras no. funcionan.

En ese contexto, empieza a abrirse paso la evaluación enraizada de y desde la gestión pública en general, apoyada -fundamentalmente- en la institucionalización de sistemas de Monitoreo y Evaluación (M\&E) orientados a aumentar la eficacia, proporcionar una mayor rendición de cuentas y transparencia en la utilización de fondos públicos (BIRF 2006). La disposición se insufla desde la banca multilateral y con el acompañamiento de agencias del sistema de la ONU, aunque destaca el mandato de los gobiernos y el impulso proveniente de la proliferación de cúpulas organizativas o redes de evaluación (de proveniencia gubernamental animadas por los organismos de cooperación internacional y/o de asociaciones de profesionales). 
En ese sentido, el Programa para la Implementación del Pilar Externo del Plan de Acción a Mediano Plazo para la efectividad en el Desarrollo (PRODEV), cuyo propósito principal es diagnosticar el nivel de institucionalización y el grado de mejora en la gestión para resultados en el desarrollo (GpRD) en los países de la región, a partir de una muestra de doce países, indica que entre 2007 y 2013 se ha registrado una evolución favorable en mejoras de la gestión pública a partir de una comprensión del sistema M\&E como una parte del ciclo de gestión. Ello asentado sobre cinco pilares: I. planificación asentada en resultados; II. Presupuesto por resultados; III. gestión financiera pública; iv. gestión de programas; v. seguimiento y evaluación. Este último -que es el menos desarrollado de los cinco- se divide en tres indicadores: monitores de la gestión gubernamental, sistemas de información estadística y evaluación de la gestión gubernamental (Kaufmann, 2016).

Una tendencia socorrida es la adopción como parámetro de la evaluación a la llamada "teoría del programa" (theory of programme), "teoría de acción" (theory of action), "ruta de cambio" (pathway of change), "motor de cambio" (engine of change), en ocasiones en su versión simplificada de "modelo lógico" (logic model), también conocido como marco lógico (logic framework). Formulaciones todas ellas al estilo de las teorías de rango medio desarrolladas y aplicadas principalmente en los Estados Unidos hace ya varias décadas (Merton 1954). Para efectos de evaluación, generalmente se entiende que se trata de una descripción de la lógica casual de cómo las intervenciones conseguirán los resultados deseados y cuáles son las secuencias de eventos necesarios para generarlos, lo que remite a la valoración de las condiciones y supuestos para que se produzca el cambio.

Por otro lado, desde la función pública en el contexto del enfoque gerencial (New Public Management) se tiende a propiciar el "incrustamiento" del ejercicio evaluativo en lo que se dado en llamar la "cadena de valor público" (CVP), principio organizador introducido por Moore (1995) como derivación del concepto pionero de Porter (1998). Bajo esta lógica la evaluación tiende a asumirse desde un sistema de gestión basado en resultados de las intervenciones públicas, en busca de evidencias para la mejora continua de desempeño de la gestión pública.

\section{Diagrama 3: Lógica de evaluación desde la Cadena de Valor Público (CVP)}

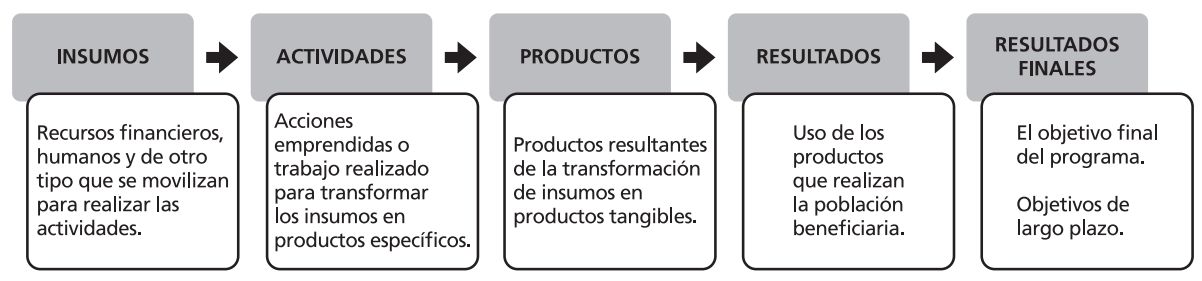

Fuente: Tomado de Banco Mundial 2011.

En América Latina y el Caribe la concreción de iniciativas de evaluación de y desde la gestión pública es muy variada. Algunos países buscan de manera intencional crear una 
agenda propia de evaluación (Chile, Costa Rica y México en buena medida), centrándose en intervenciones de interés (generalmente asumidas en forma voluntaria por parte de las instituciones); o bien se asume como un instrumento de armonización plan-presupuesto (Uruguay, República Dominicana, Perú, entre otros), en busca de recuperar una mejor articulación entre lo programado y lo ejecutado, entre algunas de las orientaciones de interés.

La experiencia pionera y más desarrollada hasta el momento, a pesar de las críticas y limitaciones, se puede atribuir al Sistema de Evaluación y Control de Gestión del Gobierno Central de Chile, iniciado en el 2000. Su propósito fundamental es mejorar la asignación y uso de recursos para contribuir a la calidad del gasto público. Las evaluaciones son realizadas por paneles de evaluadores externos. El proceso, enfocado en el Presupuesto por Resultados bajo la categoría de Presupuesto Informado, presenta como característica más sobresaliente la gradualidad en la implementación.

En ese sentido, la evaluación de programas gubernamentales se basa en la metodología del marco lógico; por tanto, se centra en aspectos de organización y gestión, y en resultados a nivel de los productos (cobertura, focalización y otros). La evaluación de impacto, al igual que la evaluación de programas nuevos, se focaliza en los resultados intermedios y finales de la población meta, construyendo estudios de línea base, utilizando modelos cuasi experimentales y acudiendo a recolección de información en terreno; en el caso de esta última perfeccionando la construcción del grupo control. Por su parte la evaluación comprehensiva del gasto analiza la consistencia estratégica entre los objetivos institucionales y sectoriales, el diseño institucional (estructura institucional y la distribución de funciones entre las distintas unidades de trabajo), los procesos productivos y de gestión, el uso de recursos financiero y los resultados en la provisión de bienes y servicios (Gobierno de Chile 2010:27-33 y 47-50).

La evaluación de y desde la gestión pública constituye una oportunidad y también un riesgo. Como ha sucedido en el pasado, las funciones requeridas, al ser incorporadas como responsabilidades del Estado, pierden protagonismo y prioridad en su atención; pasan a engrosar la lista de "buenas" intenciones en espera de asignación presupuestaria y quedan a la merced de los vaivenes de la voluntad política y/o encerradas en procesos de tecnificación de poco alcance e incidencia en la toma de decisiones. En consecuencia, uno de los retos principales es la mejora y articulación planificación-presupuestación-evaluación.

En resumen, la evaluación enraizada en y desde la gestión pública intenta generar un modelo de evaluación con prácticas y pautas centradas en:

- Intervenciones públicas en general;

- La adopción de la llamada Teoría del Cambio (u otras formas de denominación).

- La "incrustación" en llamada "cadena de valor público"(CVP). 
El patrón evaluativo a menudo recuperando prácticas y pautas de cursos de acción o recorridos anteriores, en ocasiones sin tener conciencia de ello, tiende a generar yuxtaposición de conceptualizaciones de evaluación. Su orientación principal es el diseño e instauración de diseños, sistemas o unidades de monitoreo y evaluación, con propósitos de mejoras en la eficiencia, la rendición de cuentas y la transparencia en la utilización de los fondos públicos.

\section{Reflexiones finales (¿finales?)}

En América Latina y el Caribe, especialmente en lo que transcurre del siglo xxi se podría decir que tiene una evolución altamente positiva y prometedora, especialmente en términos de la emergencia de su visibilidad y en el aprecio de sus usos.

En efecto, la evaluación pasa de un campo relativamente inexplorado a la vigencia actual y a la legitimidad social, como práctica y campo de conocimiento con aportes al estudio de la desigualdad y la pobreza, el comercio internacional, las reformas estructurales y la mejora de la administración pública en general, mostrando sensibilidad hacia la perspectiva de género, la visión intergeneracional, la sostenibilidad, entre otros ámbitos de interés.

No obstante, las tensiones teórico-metodológicas entre orientaciones y enfoques diversos, a menudo yuxtapuestos, asumidos como una especie de "moda”, en repetición acrítica de esquemas conceptuales, provenientes de contextos con características disimiles. La falta de una visión integral de enfoques de evaluación complementarios es uno de los principales vacíos de la práctica de la evaluación en estos momentos.

De una presencia prácticamente invisible en el marco del modelo de desarrollo hacia adentro vía sustitución de importaciones, la evaluación emerge en el contexto de las reformas sociales estructurales bajo la influencia de la Alianza para el Progreso, para volver a sumergirse con la crisis, la estabilización financiera y el ajuste estructural, y resurgir al amparo de la renovación del interés de los gobiernos por la política social, las reformas sectoriales, la modificación de los sistemas transversales de gestión pública y la revalorización de la función pública.

En la generalidad de los casos su presencia más destacada se deriva de la gravitación de organismos multilaterales, más que de actores nacionales. Una constante a lo largo del tiempo es la participación de la CEPAL en la profusión de literatura y asesoría a los gobiernos. En las últimas décadas se agrega la incidencia de la OECD y la CE, como también la banca multilateral en su actuación por medio del Banco Mundial, el BIRF y el BID y de las agencias del sistema de la ONU. Sin perjuicio de ello también juegan un papel importante agencias estatales, iglesias, organizaciones no gubernamentales y universidades.

En tiempos más recientes es notable la emergencia de redes de evaluación de procedencia gubernamental, animadas por la cooperación internacional y/o provenientes de asociaciones 
de profesionales y la ampliación de la oferta académica de programas universitarios de evaluación (en niveles de especialización y postgrado). Esto hace necesario el establecimiento de más y mejores vínculos de comunicación, intercambio y difusión de experiencias.

La unidad de intervención que se constituye en objeto de evaluación se mueve en forma pendular desde el proyecto en sí mismo, prácticamente como actuación única, generalmente asociado con infraestructura en apoyo al desarrollo industrial, pasando a programas amplios vinculados con reformas sociales estructurales al estilo de campañas nacionales (educación, saneamiento ambiental, agua potable, control de la natalidad, entre otras), retomando al proyecto o bien moviéndose nuevamente al programa y de vuelta al proyecto, hasta llegar en el momento actual a un marco de interés por intervenciones públicas en general, con independencia de si se trata de proyectos o programas, que busca colocar a la evaluación en el ámbito de las políticas. En la actualidad empieza a emerger -aunque todavía en forma tímida- un cierto interés por el accionar y resultados de las políticas públicas como fin último de la evaluación, en el marco del fortalecimiento de la democracia (Neirotti 2015). Una tarea pendiente es el papel de la evaluación en la articulación de la política económica y la política social y otras políticas públicas (Pichardo Muñiz 2017).

El momento privilegiado en los procesos de evaluación también se ha ido moviendo y resituándose de distintas maneras. Ubicado inicialmente en el momento ex ante (antes de la ejecución) como parte constitutiva de los estudios de factibilidad, asociado al establecimiento de prioridades de inversión y de cara al uso alternativo de recursos, pasa luego al otro extremo, a privilegiar a la evaluación ex post (una vez finalizada la ejecución), vinculada a la medición de cobertura con el propósito de valorar el alcance de las acciones. Posteriormente se va introduciendo el interés por la evaluación concurrente (durante la ejecución) para dar paso a la evaluación intermedia o de progreso y dejando prácticamente marginada a la evaluación ex ante. En ese contexto el monitoreo y seguimiento empiezan a abrirse paso. Se amplía luego el radio de acción de la evaluación, al iniciarse la incorporación, como pre requisito de la evaluación ex post, la realización de estudios línea base, para finalmente centrarse en el establecimiento de sistemas de Monitoreo y Evaluación como parte de los intentos de institucionalización de la evaluación de y desde la gestión pública. En la actualidad resulta necesario aunar esfuerzos hacia un enfoque integrador de la evaluación en sus distintos momentos, que otorgue prioridad a la evaluación ex ante como criterio para alimentar y retroalimentar a la toma de decisiones institucionales, incorpore al seguimiento y se diferencie de otras funciones conexas (como el control).

La evaluación muestra asimismo adelantos notables de la concepción inicial de la participación de la población destinataria en tanto fuente de información, pasando de la consulta de opiniones calificadas especialmente cuando de medición de la satisfacción de servicios se trata, a la medición de impactos, entendidos como cambios en la 
situación, condición y calidad de vida. Del mismo modo, hace falta avanzar en la evaluación de impactos en las instituciones, en las organizaciones y en la sociedad en su conjunto, asumido esto como perspectiva de evaluación en el marco de una función ética y no únicamente como un criterio más de evaluación,. Colocándola como proceso de aprendizaje de cara a la construcción del futuro, justo en el lugar que le corresponde.

El giro más notable en los distintos recorridos de la evaluación en América Latina y el Caribe es, sin duda, el observado en la orientación predominante en el uso de los indicadores, desde un instrumental proveniente en lo fundamental de la disciplina económica a un instrumental más amplio que se nutre de la gestión y gerencia y de otras disciplinas. Así, la perspectiva inicial anclada a indicadores como la Tasa Interna de Retorno (TIR), el Valor Neto Actualizado (VAN) y otros indicadores financieros, va quedando atrás. Primero "arropada" por indicadores relativos al volumen de trabajo, productos o servicios y realizaciones, desde la visión convencional de la administración. Luego en procura de medidas de estadística descriptiva, para realizar valoraciones en términos cuantitativos. Paulatinamente se introduce el interés de la evaluación de la calidad, con base en estándares nacionales e internacionales, especialmente en el marco de procesos de certificación (industrial, ambiental o de otra naturaleza) y acreditación (principalmente universitaria). Posteriormente, aparece un marcado énfasis en el uso de instrumental estadístico más sofisticado y modelos econométricos, el diseño y aplicación de modelos experimentales y cuasi experimentales con o sin grupo de control. Más recientemente, y en algunos casos en forma concomitante al interés anterior, la batería de indicadores tiende a fijarse con base en la formulación del marco lógico, asumida como versión simplificada de la Teoría del Cambio.

Es necesario colocar cada uno de estos enfoques de indicadores en el lugar que le corresponde en la evaluación, buscando las debidas articulaciones y complementariedades, retomando esfuerzos importantes como la perspectiva de indicadores de Estado-PresiónRespuesta (EPR), el análisis de múltiples criterios, la valoración socioeconómica ambiental y otros de naturaleza similar o complementaria (Pichardo Muñiz 2008en prensa). 
Tabla 2: Cursos de acción o recorridos de la evaluación en América Latina y el Caribe

\begin{tabular}{|c|c|c|c|c|c|}
\hline $\begin{array}{l}\text { Cursos de } \\
\text { Acción o } \\
\text { Recorridos/ } \\
\text { Criterios } \\
\text { para su } \\
\text { caracterización }\end{array}$ & $\begin{array}{l}\text { Decisiones } \\
\text { políticas de } \\
\text { envergadura } \\
\text { global con } \\
\text { impacto en la } \\
\text { evaluación }\end{array}$ & $\begin{array}{l}\text { Incidencia de } \\
\text { organismos } \\
\text { internacionales }\end{array}$ & $\begin{array}{l}\text { Unidad de } \\
\text { intervención } \\
\text { objeto de } \\
\text { evaluación }\end{array}$ & $\begin{array}{l}\text { Momento de } \\
\text { evaluación } \\
\text { privilegiado }\end{array}$ & $\begin{array}{l}\text { Orientación } \\
\text { predominante } \\
\text { en el uso de los } \\
\text { indicadores }\end{array}$ \\
\hline $\begin{array}{l}\text { Evaluación } \\
\text { derivada de las } \\
\text { primeras iniciativas } \\
\text { de planificación } \\
\text { nacional bajo la } \\
\text { influencia de la } \\
\text { CEPAL. }\end{array}$ & $\begin{array}{l}\text { Modelo de } \\
\text { desarrollo hacia } \\
\text { adentro vía } \\
\text { sustitución de } \\
\text { importaciones. }\end{array}$ & CEPAL e ILPES. & $\begin{array}{l}\text { Proyecto de } \\
\text { Infraestruc- } \\
\text { tura en apoyo } \\
\text { a la política } \\
\text { de desarrollo } \\
\text { industrial. }\end{array}$ & $\begin{array}{l}\text { Ex ante (antes } \\
\text { de la ejecución): } \\
\text { Estudios de } \\
\text { Factibilidad. }\end{array}$ & $\begin{array}{l}\text { Económica-financiera } \\
\text { (TIR,VAN y } \\
\text { otros indicadores } \\
\text { financieros) }\end{array}$ \\
\hline $\begin{array}{l}\text { Evaluación } \\
\text { asociada con } \\
\text { experiencias de } \\
\text { planificación } \\
\text { nacional al calor } \\
\text { de la Alianza para } \\
\text { el Progreso. }\end{array}$ & $\begin{array}{l}\text { Propuestas de } \\
\text { reformas sociales } \\
\text { estructurales. }\end{array}$ & $\begin{array}{l}\text { Alianza para el } \\
\text { Progreso. }\end{array}$ & $\begin{array}{l}\text { Programas en } \\
\text { el marco de } \\
\text { las reformas } \\
\text { sociales (en } \\
\text { particular } \\
\text { campañas } \\
\text { nacionales). }\end{array}$ & $\begin{array}{l}\text { Ex post } \\
\text { (finalizada la } \\
\text { ejecución). }\end{array}$ & $\begin{array}{l}\text { Administración } \\
\text { (Volumen de trabajo, } \\
\text { Productos o Servicios } \\
\text { y Realizaciones). }\end{array}$ \\
\hline $\begin{array}{l}\text { Evaluación } \\
\text { inspirada } \\
\text { en la Ayuda } \\
\text { Humanitaria. }\end{array}$ & $\begin{array}{l}\text { Debilitamiento } \\
\text { y descrédito del } \\
\text { Estado } \\
\text { Preponderancia } \\
\text { de la Sociedad } \\
\text { Civil Conflictos } \\
\text { bélicos. }\end{array}$ & $\begin{array}{l}\text { Agencias de } \\
\text { Estado, } \\
\text { Universidades, } \\
\text { Iglesias, y otras } \\
\text { Organizaciones No } \\
\text { Gubernamentales } \\
\text { (ONG). }\end{array}$ & Proyecto. & $\begin{array}{l}\text { Evaluación } \\
\text { Concurrente } \\
\text { (durante la } \\
\text { ejecución): } \\
\text { Evaluación } \\
\text { Intermedia } \\
\text { o de Progreso. }\end{array}$ & $\begin{array}{l}\text { Estadística descriptiva } \\
\text { (comparación entre } \\
\text { lo programado y } \\
\text { lo efectivamente } \\
\text { ejecutado). }\end{array}$ \\
\hline $\begin{array}{l}\text { Evaluación } \\
\text { encauzada por la } \\
\text { Cooperación para } \\
\text { el Desarrollo. }\end{array}$ & $\begin{array}{l}\text { Reforma y } \\
\text { Moderniza-ción } \\
\text { del Estado. }\end{array}$ & $\begin{array}{l}\text { OECD } \\
\mathrm{CE}\end{array}$ & $\begin{array}{l}\text { Programa } \\
\text { (aunque } \\
\text { también } \\
\text { proyecto). }\end{array}$ & $\begin{array}{l}\text { Evaluación } \\
\text { Concurrente. } \\
\text { Monitoreo y } \\
\text { Seguimiento. }\end{array}$ & $\begin{array}{l}\text { Gestión y Gerencia } \\
\text { (Estándares nacionales } \\
\text { e internacionales). }\end{array}$ \\
\hline $\begin{array}{l}\text { Evaluación bajo } \\
\text { el alero de la } \\
\text { Protección Social. }\end{array}$ & $\begin{array}{l}\text { Renovación del } \\
\text { interés de los } \\
\text { gobiernos por la } \\
\text { Política Social. }\end{array}$ & $\begin{array}{l}\text { Banco Mundial } \\
\text { BIRF } \\
\text { BID } \\
\text { Agencias del } \\
\text { Sistema de la } \\
\text { ONU }\end{array}$ & $\begin{array}{l}\text { Programa (con } \\
\text { cierto nivel de } \\
\text { complejidad). }\end{array}$ & $\begin{array}{l}\text { Estudio de Línea } \\
\text { Base Evaluación } \\
\text { Ex Post. }\end{array}$ & $\begin{array}{l}\text { Instrumental } \\
\text { estadístico sofisticado } \\
\text { y modelos } \\
\text { econométricos } \\
\text { Modelos } \\
\text { experimentales y cuasi } \\
\text { experimentales con o } \\
\text { sin grupo de control. }\end{array}$ \\
\hline $\begin{array}{l}\text { Evaluación de y } \\
\text { desde la gestión } \\
\text { pública. }\end{array}$ & $\begin{array}{l}\text { Reformas } \\
\text { sectoriales, } \\
\text { modificación } \\
\text { de los sistemas } \\
\text { transversales } \\
\text { de la gestión } \\
\text { pública y } \\
\text { revalorización } \\
\text { de la función } \\
\text { pública. }\end{array}$ & $\begin{array}{l}\text { Banca Multilateral } \\
\text { Agencias del } \\
\text { Sistema de la } \\
\text { ONU } \\
\text { Gobiernos } \\
\text { Universidades } \\
\text { Redes de } \\
\text { profesionales en } \\
\text { evaluación. }\end{array}$ & $\begin{array}{l}\text { Intervenciones } \\
\text { públicas en } \\
\text { general. }\end{array}$ & $\begin{array}{l}\text { Sistemas de } \\
\text { Monitoreo \& } \\
\text { Evaluación. }\end{array}$ & $\begin{array}{l}\text { Gestión y Gerencia } \\
\text { (Cadena de Valor } \\
\text { Público) }\end{array}$ \\
\hline
\end{tabular}

Fuente: Elaboración propia con base en el texto desarrollado en el artículo

Resumiendo, se puede decir que en América Latina y el Caribe, sin duda alguna, los usos de la evaluación se han ido ampliado y fomentado, como puede apreciarse en la producción de literatura, en los programas de estudios, en la proliferación de eventos, en la integración de profesionales de las más diversas disciplinas de estudio, en la cantidad de iniciativas de evaluación, en el desarrollo de redes y otras instancias organizativas y en la creciente especialización 
de un "mercado" de evaluación, con creciente demanda desde las instituciones del sector público y de las capacidades de oferta profesional. Se ha pasado desde un interés principalmente asociado al destino de la inversión pública o de los fondos de la ayuda o cooperación internacional a un creciente interés ¿real? de los gobiernos, las universidades y las asociaciones profesionales que invita a gestores, ejecutores, población destinaria, poder legislativo y grupos de opinión en general a participar y a tener conciencia de la importancia y pertinencia de la evaluación en la mejora de la toma de decisiones institucionales, organizativas y nacionales. Pese a que los supuestos iniciales (linealidad y control axiológico), herencia de la investigación social, permanecen prácticamente inalterados (Pichardo Muñiz 2017).

Hablar hoy en día de evaluación está de moda, junto a la política pública y al Estado. Sin embargo muchos vacíos están presentes, en particular las formas de entenderla y practicarla desde la complejidad del mundo de hoy: una tarea pendiente, apenas iniciada. América Latina y el Caribe cuentan con una extraordinaria capacidad para aportar en esa tarea. En particular, porque la evaluación se desarrolla en y desde la práctica. No obstante, el gran reto es descolonizar los métodos de evaluación.

El desafío está en dar el salto de la evaluación que genera temor, porque busca control; desde propósitos de mejoras en la eficacia, la rendición de cuentas y la transparencia en la utilización de los fondos públicos, a iniciativas de intervención que den muestras de procesos globales y sostenibles como expresión de una cultura de evaluación en el marco del fortalecimiento y profundización de los espacios democráticos, en procura de mejoras sostenibles en la calidad de vida humana, pues al fin de cuentas de lo que se trata es que ahora que la gente vive más, viva mejor. 
${ }^{1}$ Este artículo forma parte de un proyecto de investigación más amplio que se desarrolla bajo la coordinación de la autora, con el título de "Acercando la Política Económica y la Política Social: el papel de la evaluación”. Comentarios y sugerencias son bien recibidos por medio del correo indicado.

${ }^{2}$ Tanto los recorridos como la periodización son indicativas y no necesariamente tienen que coincidir con algún país en particular o situación específica. 


\section{Referencias bibliográficas}

Banco Mundial (2011). La evaluación de impacto en la práctica. Washington D.C.: Banco Internacional de Reconstrucción y Fomento/Banco Mundial.

IRF (Banco Internacional de Reconstrucción y Fomento) / Banco Mundial (2006). Hacia la Institucionalización de los Sistemas de Monitoreo y Evaluación en América Latina y el Caribe. Actas de una Conferencia del Banco Mundial/Banco Interamericano de Desarrollo. Washington D.C. Banco Mundial y Banco Interamericano de Desarrollo.

Bauer, Raymond (comp.) (1966) Social Indicators. Cambridge, Massachusetts, The MIT Press

Campbell, Douglas y Stanley, Julian (1963). Experimental and Quasi-Experimental Designs for Research. Chicago: Rand McNally.

Capra, Fritjof (1998). La trama de la vida: Una nueva perspectiva de los sistemas vivos Barcelona: Editorial Anagrama.

Cardoso, Fernando Henrique (1977). "La originalidad de la copia: la CEPAL y la idea de desarrollo". En Revista de la CEPAL No4, 7-40.

Cohen, Ernesto y Rolando Franco (1992). Evaluación de Proyectos Sociales. México D.F., Siglo XXI Editores.

CEPAL, Comisión Económica para America Latina y el Caribe (1958). Manual de Proyectos de Desarrollo Económico. Estudio preparado por el Programa CEPAL/AAT de capacitación en material de desarrollo económico. México D.F.: Naciones Unidas.

Comisión Europea (2002). Guía Gestión del Ciclo de Proyecto. Preparada por PARTICIP $\mathrm{GmbH}$, Freiburg, Alemania, en colaboración con FTP International Ltda, Bruselas, Bélgica.Versión 2.0.

Cook, Thoas D y Campbell, Douglas (1979). Quasi Experimentation: Design and Analysis for Fields Settings. Chicago: Rand McNally.

Fontaine, Guillaume (2015). El análisis de politicas públicas: Conceptos, teorías y métodos. FLACSO Ecuador. Grupo Editorial Siglo XXI editores. Barcelona, España, Anthropos editorial. 
Gobierno de Chile. Ministerio de Hacienda. Dirección de Presupuesto (2010). Presupuesto por Resultados y la Consolidación del Sistema de Evaluación y Control de Gestión del Gobierno Central. Arenas de Mesa, A. y Berner Herrera, H. División de Control de Gestión.

ILPES, Instituto Latinoamericano de Planificación Social (1973). Guía para la presentación de proyectos. México D.F., Siglo XXI editores.

Kaufmann, Jorge (2016). "Experiencias recientes de fortalecimiento de los sistemas de monitoreo y evaluación en América Latina y el Caribe”. En Claudia M. Pasquetti y Carmen Salas, eds., Los Sistemas de Monitoreo y Evaluación: Hacia la mejora continua de la planificación estratégica y la gestión pública. Memorias de la IX Conferencia Internacional de la Red Latinoamericana y del Caribe de Monitoreo y Evaluación y del I Seminario Internacional de Seguimiento y Evaluación, Lima: BID-Banco Mundial-CEPLAN, 52-59.

Merton, Robert (1954). Teoría y estructuras sociales. México: Fondo de Cultura Económica. (Edición original en inglés, 1949).

Moore, Mark (1995). Creating Public Value. Strategic Management in Goverment. Cambridge, Ma.: Harvard University Press.

Neirotti, Nerio (2012) "Evaluation in Latin America: Paradigms and Practices". En S. Kushner \& S. Rotondo (eds.) Evaluation voices from Latin American. News Directions for Evaluation. Washington, DC:American Evaluation Association, No 134:7-16.

Neirotti, Nerio, coord. (2015): La Evaluación de las Políticas Públicas. Reflexiones y experiencias en el escenario actual de transformaciones del Estado. En colaboración con María Eugenia Brissón y Matías Mattalini (Editores). Universidad Nacional de Lanús (UNLa). Colección Políticas Públicas. Ediciones de la UNLa.

Pichardo Muñiz, Arlette (1984) Planificación y Programación Social: Bases para el diagnóstico y la formulación de Programas y Proyectos Sociales. San José, Costa Rica: Editorial de la Universidad de Costa Rica.

Pichardo Muñiz, Arlette (1993). Evaluación del Impacto Social: el valor de lo humano ante la crisis y el ajuste. Reimpresión 1997. Buenos Aires: Editorial Hvmanitas.

Pichardo Muñiz, Arlette (2013). "Advances in Strategic Planning of the Public Sector in Latin American and the Caribbean: Balance and perspectives”. En Quality Issues in the 21th Century, Vol. 2, and No1:1-17. 
Pichardo Muñiz, Arlette (2014) "Los programas de transferencias monetarias condicionadas en América Latina y el Caribe: un nuevo ¿rostro? de la política social”. En Cuadernos de Política Económica 1-2014. Heredia, Costa Rica: Centro Internacional en Política Económica para el Desarrollo Sostenible (CINPE). Universidad Nacional (UNA).

Pichardo Muñiz, Arlette (2017) Ábreme la puerta. Casa de Aprendiencia de la Política Social y otras políticas. Santo Domingo, República Dominicana: Editora Búho.

Pichardo Muñiz, Arlette (2008 en prensa) "La Política Social en América Latina y el Caribe". En Políticas Económicas para el Desarrollo Sostenible. Libro conmemorativo del XX aniversario del Centro Internacional de Política Económica para el Desarrollo Sostenible (CINPE). Editores Jeffrey Orozco Barrantes, Olman Segura Bonilla y Suyen Alonso Ubieta. Rockville, Md.: Global South Press. 255-286.

Pichardo Muñiz, Arlette (en prensa) La evaluación frente a los Objetivos del Desarrollo Sostenible (ODS).

Pichardo Muñiz, Arlette y Hélio Fallas Venegas (2014). “Acercando la Política Económica y la Política Social: el papel de la Evaluación”. En Encuentro Internacional Acercando la Política Económica y la Política Social: el papel de la EVALUACIÓN. Memoria. Heredia, Costa Rica: CINPE-SPE-FOCEVAL. Págs. 13-50.

Porter, Michael (1998). Competitive Advantage: Creativity and Sustaining of Superior performance. Cambridge, Ma.: Harvard Business School Press.

Prebisch, Raúl (1963). Hacia una dinámica del desarrollo latinoamericano. México: Fondo de Cultura Económica.

Real Academia Española (2017) Diccionario de la lengua española. Consultado en http://dle.rae.es/?id=M4SlkEr).

Weiss, Carol (1978): Investigación evaluativa: Métodos para determinar la eficiencia de los programas de acción. México D.F., Editorial Trillas (ed. original en inglés, 1972).

\section{Cómo citar este artículo:}

Pichardo Muñiz, Arlette (2019) "La Evaluación. Una mirada desde América Latina y el Caribe". Revista Perspectivas de Políticas Públicas vol. 8 Nº16: 441-468 